\title{
Diffuse Optical Tomography based on time-resolved compressive sensing
}

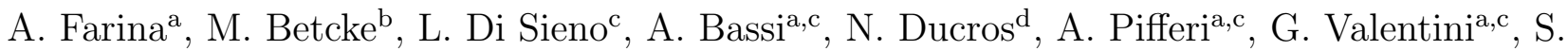 \\ Arridge $^{\mathrm{b}}$, and C. D'Andrea ${ }^{\mathrm{c}, \mathrm{e}}$ \\ ${ }^{a}$ Consiglio Nazionale delle Ricerche, IFN, Piazza L. da Vinci 32, 20133 Milan, Italy \\ ${ }^{\mathrm{b}}$ Centre for Medical Image Computing, University College London, Malet Place, London \\ WC1E 6BT, United Kingdom

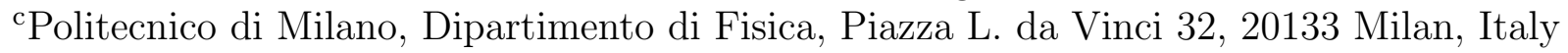 \\ ${ }^{\mathrm{d}}$ CREATIS, CNRS UMR5220, INSERM U1044, Universit de Lyon, INSA Lyon, Villeurbanne, \\ France \\ ${ }^{\mathrm{e}}$ Center for Nano Science and Technology@PoliMi, Istituto Italiano di Tecnologia, via Giovanni \\ Pascoli 70/3, 20133 Milan, Italy
}

\begin{abstract}
Diffuse Optical Tomography (DOT) can be described as a highly multidimensional problem generating a huge data set with long acquisition/computational times. Biological tissue behaves as a low pass filter in the spatial frequency domain, hence compressive sensing approaches, based on both patterned illumination and detection, are useful to reduce the data set while preserving the information content. In this work, a multiple-view time-domain compressed sensing DOT system is presented and experimentally validated on non-planar tissue-mimicking phantoms containing absorbing inclusions.
\end{abstract}

Keywords: Time-resolved, diffuse optical tomography, compressive sensing, single pixel camera

\section{INTRODUCTION}

Diffuse Optical Tomography (DOT) is an optical imaging technique which aims at quantitatively 3D reconstructing optical parameters (absorption and scattering coefficient) inside in vivo macroscopic organisms ranging from small animals (e.g. mice) to humans. In particular, the absorption coefficient is related to tissue chromophores (e.g. water, lipid, oxy- and deoxy-haemoglobin), while the scattering coefficient provides information about internal structure. The quantification of these parameters and their spatial distribution provides important anatomical and functional features of the biological tissue useful for both diagnostic purposes (e.g. tumor detection $)^{1}$ and functional information (e.g. brain functional studies). ${ }^{2}$ Closely related to DOT is Fluorescence Molecular Tomography (FMT) which aims to quantify and localize selective fluorescent markers designed (exogeneous) or identified (endogeneous) to target molecular processes. ${ }^{3,4}$ FMT is related to DOT both in terms of technical issues of the system (e.g. similar measurement scheme) and because DOT can be considered as a prerequisite to improve quantification and localization of fluorescent markers.

The usual scheme of a DOT system is based on a point light beam (source) raster scanned around the sample. For each injection point, the diffused light exiting the sample is measured either by a scanning detector or a parallel detector (e.g. CCD, CMOS, Gated camera). Then, by solving an inverse problem, the optical parameters' distribution inside the tissue can be reconstructed. In general a single detector has higher performances in terms of spectral sensitivity (e.g. Near Infrared range) and temporal resolution compared with a parallel detector. Hence, information content is maximized by adopting a scanning system in both illumination and detection space. Despite the high information content, this scheme shows significant disadvantages because of the huge data set originating from the large number of source-detector combinations. This leads to extremely long acquisition and

Further author information: (Send correspondence to Cosimo D'Andrea)

E-mail: cosimo.dandrea@polimi.it, Telephone: +39-02-2399-6114

Optical Tomography and Spectroscopy of Tissue XII, edited by Bruce J. Tromberg, Arjun G. Yodh,

Eva Marie Sevick-Muraca, Robert R. Alfano, Proc. of SPIE Vol. 10059, 100590I · @ 2017 SPIE

CCC code: $1605-7422 / 17 / \$ 18 \cdot$ doi: $10.1117 / 12.2252594$ 
computational times which are generally not compatible with pre-clinical and clinical environments. The long acquisition time originates both from the high number of measurements and from safety rules which impose a limitation on the light density at each point and, therefore, a longer acquisition time for each measurement. Moreover the huge data set implies long computational times which rapidly become intractable with standard computers.

The dimension of the data set is further complicated by the highly multidimensional features of DOT scheme. In fact, apart from the measurement of the diffused light intensity around the sample, other parameters provide useful information such as adopting a multiple views acquisition scheme $e^{5,6}$ and the measurement of temporal and spectral profiles. ${ }^{7,8}$ A multiple views scheme consists of measuring the diffused light at different sample rotation which is crucial to improve the spatial resolution. Time-resolved measurements allows one to better disentangle absorption from scattering coefficient, ${ }^{9}$ to evaluate the depth of the inclusion ${ }^{10}$ and to estimate fluorescence lifetime of fluorochromes in the case of FMT. Spectral information of the diffused light is mainly exploited to better discriminate the different chromophores inside the tissue.

In the last years novel illumination schemes based on structured illumination have been proposed. ${ }^{8,11,12}$ The possibility to use a wide field illumination, in contrast to a single source, allows one to input higher intensity on the sample without exceeding the light density imposed by safety limits. Moreover a turbid medium is characterized by a low bandwidth of the spatial information content. This allows one to illuminate the sample with a limited number of patterns and, therefore, to preserve the information content while reducing the data set.

Recently the same approach has been adopted on the detection side. If a parallel detector is used, this approach leads to a post-processing reduction of the data set by exploiting the low pass behavior of the spatial information in a scattering medium. ${ }^{13,14}$ Moreover, by adopting the Single Pixel Camera (SPC) scheme, ${ }^{15}$ a patterned detection can be exploited at the acquisition stage. The basic idea is to project the image of the object (e.g. the diffused light exiting the sample) on a spatial modulator and to collect the light integral by a single pixel detector. By repeating the measurement with different patterns a sampling in the spatial frequency domain (rather than raster scanning) can be carried out. Similarly to structured illumination, a limited number of patterns can be adopted, exploiting the low bandwidth of the scattering medium. It is worth mentioning that a SPC detection scheme has important instrumental advantages, such as the use of a single pixel detector with higher performances (e.g. temporal resolution and spectral coverage) with respect to parallel ones.

The use of structured illumination/detection allows a reduction of the data set while preserving the information content and reducing the acquisition and computational times. ${ }^{16}$ Moreover, the use of a wide field approach allows from the first measurements a global view, even if at low spatial resolution, which is particularly suited towards an adaptive approach. Finally it is worth stressing a simplification of the system and a reduction of the cost, mainly given by SPC approach.

In this work a time-resolved DOT system with multiple views acquisition has been experimentally realized and validated. Measurements on cylindrical tissue mimicking phantoms with absorption inclusions have been carried out in order to test the imaging and tomographic capability of the system, obtaining state of the art reconstruction.

\section{MATERIALS AND METHODS}

The experimental set-up is shown in Fig. 1. A supercontinuum laser source (SuperK Extreme, NKT) provides light pulses (pulse width of tens of picoseconds) at the repetition rate of about $80 \mathrm{MHz}$ over a broad spectral range (450-2000 nm). The supercontinuum is spectrally filtered by means of an acousto-optic tunable filter (AOTF) to select light pulses at $650 \mathrm{~nm}$ with $5 \mathrm{~nm}$ bandwidth. Through an optical fiber, the light is coupled to a Total Internal Reflection prism and then to a Digital Micromirror Device (DMD Discovery kit 1100, Vialux, Germany) which allows to spatially modulate the light. The pulsed structured light is, finally, imaged over an area of $3 \times 3 \mathrm{~cm}^{2}$ of the sample by means of a lens $(\mathrm{f}=50 \mathrm{~cm})$. The sample is placed on a rotational stage in order to allow multiple-view acquisition. By means of a lens $(\mathrm{f}=60 \mathrm{~cm})$ the diffused light, exiting the sample, over an area of about $2 \times 2 \mathrm{~cm}^{2}$, is imaged on a second DMD (DMD Discovery 4100, Vialux). The light reflected by the second DMD is focused by a long working-distance objective $(10 \mathrm{X} / 0.25)$ into a $1 \mathrm{~mm}$ diameter step-index 
optical fiber. A photomultiplier (PMT) (HPM-100-50, Becker \& Hickl) measures the light exiting the fiber and, by means of a Time-Correlated Single Photon Counting (TCSPC) board the temporal profile of the diffuse light is recorded. The Instrumental Response Function (IRF), taking into account the detector time-response and fiber broadening is about 250 ps FWHM.

The time-resolved compression on the detection is achieved by repeating the measurements with different patterns loaded on the second DMD. By means of a flip mirror, a Continuous Wave (CW) low noise 16-bit cooled CCD camera (Versarray 512, Princeton Instruments) is placed on the detection side for direct imaging of the sample output plane. This direct imaging is achieved by rotating all the DMD's pixels in the same state. The system is fully computer controlled by a home-made LabView software that allows one to automatically acquire the whole data set such as the illumination/detection patterns, sample rotation and time acquisition. Measurements were carried out on a homogeneous cylindrical phantom $(\varnothing=20 \mathrm{~mm}$, height $50 \mathrm{~mm})$ made of

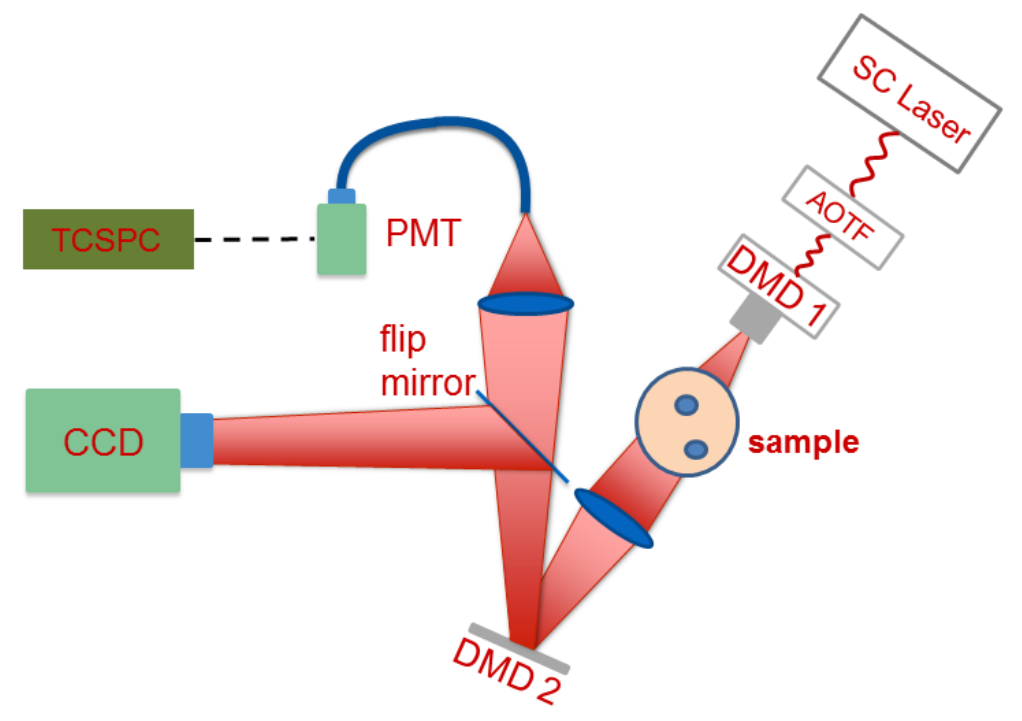

Figure 1. Experimental set-up

epoxy resin, $\mathrm{TiO}_{2}$ (as scatterer) and toner (as absorber) mimicking the optical properties of biological tissues. The optical parameters of the phantom were measured by means of a broadband time-resolved spectroscopy system $^{17}$ obtaining the following values: absorption coefficient $\left(\mu_{a}\right)$ about $0.01 \mathrm{~mm}^{-1}$ and reduced scattering coefficient $\left(\mu_{s}^{\prime}\right)$ about $1 \mathrm{~mm}^{-1}$. Two holes (see Fig. 2) were drilled into the sample $(\varnothing=1.6 \mathrm{~mm})$ in order to allow us to insert inclusions with different optical parameters. In particular a totally absorbing solid rod has been exploited in this work.

In order to acquire the shape of the phantom, and consequently to create its mesh, 360 shadows of the sample (every $1^{\circ}$ ) have been acquired. ${ }^{6}$ Before starting with the measurement, a calibration procedure has to be performed to register input/output patterns on the sample, and it is worth stressing its importance to improve the quality of the tomographic reconstruction. To register the patterns, a mapping function of the source and detector points on the corresponding DMD has to be experimentally derived. This step is quite critical to improve the accuracy of the forward model needed by the tomographic reconstruction. By means of the CCD camera, images on the detection side have been acquired, allowing to register the detection area over the sample. 
Similarly, images of the illumination side have been acquired with a specific low-cost camera positioned for this specific purposes in order to register the illumination area over the sample. This procedure further allows one to map the single element of the DMD to the illumination/detection point on the sample.

The Single-Pixel Camera hardware performs the following operation:

$$
c_{k}(t)=\iint I(x, y, t) h_{k}(x, y,) d x d y
$$

where $c_{k}(t)$ is the time-resolved profile measured by the PMT, $I(x, y, t)$ the time-resolved image on the output plane of the sample and $h_{k}(x, y)$ is the $\mathrm{k}$-th pattern loaded on the DMD, typically belonging to an orthonormal basis set. In this work we used Walsh-Hadamard (WH) patterns, for both illumination and detection, because, due to the fact that they are binary, they can be easily realized on a DMD and, moreover, they form a complete basis with finite cardinality. Mathematically, N-th order (N power of 2) WH patterns can be described by NxN squared matrixes $H_{k}$ composed only by +1 and -1 elements. Hereafter an example of order $2 \mathrm{WH}$ matrix:

$$
H_{1}=\frac{1}{N^{2}}\left[\begin{array}{ll}
1 & 1 \\
1 & 1
\end{array}\right], H_{2}=\frac{1}{N^{2}}\left[\begin{array}{ll}
1 & -1 \\
1 & -1
\end{array}\right], H_{3}=\frac{1}{N^{2}}\left[\begin{array}{ll}
-1 & 1 \\
-1 & 1
\end{array}\right], H_{4}=\frac{1}{N^{2}}\left[\begin{array}{cc}
1 & -1 \\
-1 & 1
\end{array}\right]
$$

In order to physically realize these patterns, for each of them two were created composed with 0 and 1 so that the difference gives the true WH pattern. Besides this operation increases the total acquisition time, it is very helpful for noise subtraction.

It is worth noting that due to the orthonormality property of $H_{k}$, spatially realized by the pattern $h_{k}(x, y)$, it is possible to reconstruct an $\mathrm{N}$-th order approximation $\hat{I}(x, y, t)$ of the time-resolved image $I(x, y, t)$ using the following backprojection operation (fast Walsh-Hadamard inverse transform $^{18}$ ):

$$
\hat{I}(x, y, t)=\sum_{k=1}^{N^{2}} c_{k}(t) h_{k}(x, y, t)
$$

\subsection{Tomographic reconstruction}

The tomographic reconstruction algorithm relies on the minimization of an objective function $\Psi(\mathbf{x})$ composed by the sum of a quadratic term and a penalty function for the regularization:

$$
\Psi(\mathbf{x})=\frac{1}{2} \sum_{n}\left[\frac{y_{n}-f_{n}(\mathbf{x})}{\sigma_{n}}\right]^{2}+\tau R(\mathbf{x})
$$

where $\mathbf{x}$ represents the variables vector (absorption coefficient for every voxel), $y_{n}$ is the measurement, $f_{n}(\mathbf{x})$ is the forward model, $\sigma$ is a scaling factor given, in this case, by the forward model with homogeneous optical properties. $R(\mathbf{x})$ is the regularization functional given in this case by the Total Variation (TV) and $\tau$ is the hyperparameter giving the weight of the regularization functional. Minimization of $\Psi$ is achieved by a 3 iterations damped Gauss-Newton method followed by a line-search algorithm. ${ }^{19}$ The damped Gauss-Newton method requires the calculation of the Jacobian matrix that, for the time-domain diffusion equation, results:

$$
\left.\frac{\partial f}{\partial \mu_{a}}\right|_{\mathbf{r}, t}=m(t) \otimes G(\mathbf{r}, t) \otimes G^{\star}(\mathbf{r}, t)=\int_{0}^{t} \int_{0}^{t^{\prime \prime}} m\left(t^{\prime}\right) G\left(\mathbf{r}, t^{\prime \prime}-t^{\prime}\right) G^{\star}\left(\mathbf{r}, t-t^{\prime \prime}\right) d t^{\prime} d t^{\prime \prime}
$$

where $m(t)$ is the IRF, $G(\mathbf{r}, t)$ is the fluence distribution in the sample generated by the sources and $G^{\star}(\mathbf{r}, t)$ is the adjoint fluence when sources are placed in the detector positions. This operation is very time- and memoryconsuming thus, to speed it up, the convolutions are performed via Fast Fourier Transform (FFT) in time. The only drawback of this operation is that the memory needed quadruples with respect to performing it in time because a zero-padding is needed to avoid aliasing and also because the FFT is complex-valued. After that, all the values belonging at the early(late)-gate used are summed up and stored in the final matrix. 


\section{RESULTS AND DISCUSSION}

In order to demonstrate the tomographic capability of the time-resolved DOT system, reconstructions by using one temporal gate (early or late) of the time-resolved temporal curve have been carried out. Early gate is obtained by integrating the temporal profile over a width of 300 ps from $1 \%$ to $80 \%$ of the peak intensity on the rising edge, while late gate width is about 860 ps ranging from $80 \%$ to $1 \%$ of the peak intensity on the falling edge. Measurements were carried out for 8 different views at step of $45^{\circ}$ to cover a complete rotation of the sample, and for each view, a squared illumination pattern and 8x8 WH detection patterns have been adopted. This leads to $127 \mathrm{WH}$ patterns for each view. Acquisition time for each pattern is $1 \mathrm{~s}$. The mesh used for the forward problem has 108000 elements and 1016 temporal points (sampled in 130 temporal steps of 12 ps width) have been generated. The computational time for the forward problem is about $25 \mathrm{~s}$ on a machine mounting a $2.5 \mathrm{GHz}$ quad-cores Intel i7 processor and $16 \mathrm{~Gb}$ RAM memory. The Jacobian computational time in our case is about 1.5 hours. The overall reconstruction time is about 5 hours.

In Fig. 2 the tomographic reconstruction, by using the early gate, of the absorption coefficient $\mu_{a}$ at different vertical slices is reported. Only the upper half of the cylinder can be reconstructed due to the illumination and detection area. A good reconstruction quality is obtained concerning both the localization and relative contrast of the two inclusions. Fig. 2 also shows an example of line profile across the inclusions at plane $z=17.5 \mathrm{~mm}$ for both reconstruction with early and late gate. As expected, the localization and shape characterization of the inclusions improves by using the early gate.
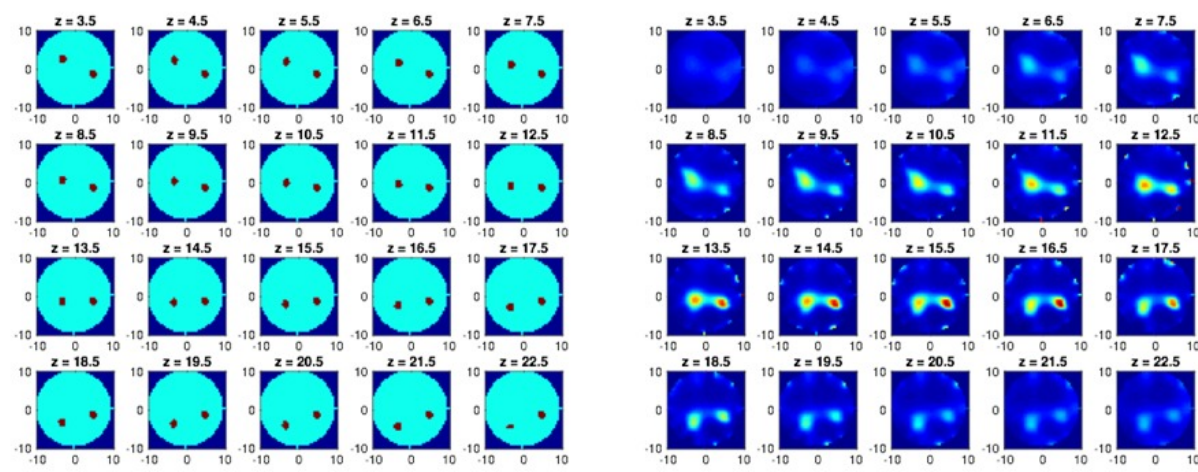

(a)

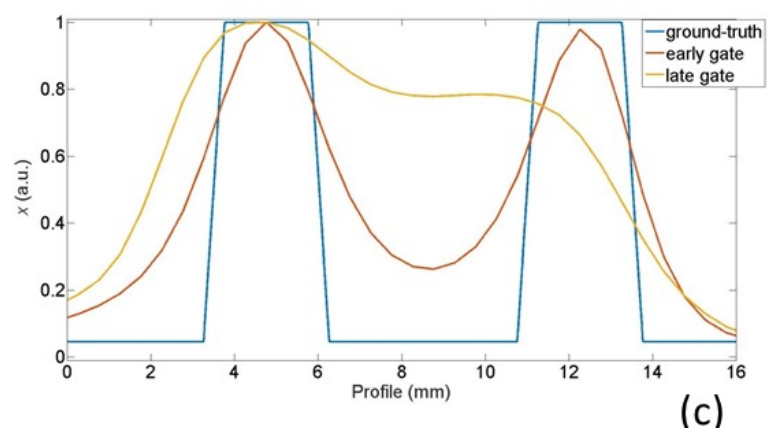

(b)

Figure 2. Reconstruction of the absorption coefficient using an early gate. Slices, at step of $1 \mathrm{~mm}$, are displayed from $z=22.5 \mathrm{~mm}$ (top of the cylinder) to $z=3.5 \mathrm{~mm}$. The middle of the cylinder corresponds to $z=0 \mathrm{~mm}$ plane. (a) groundtruth; (b) reconstruction (values are in $\mathrm{mm}^{-1}$ );(c) Profile across a line connecting the two inclusions centers on a plane at $z=17.5 \mathrm{~mm}$ for both early (red line) and late (yellow line) gate reconstructions.

\section{CONCLUSIONS}

In conclusion, in this work, a time-resolved DOT system based on structured illumination, single pixel camera detection and multiple views acquisition has been experimentally realized and validated on a tissue-mimicking 
cylindrical phantom, obtaining a state of the art reconstruction quality. Future work will be addressed towards an optimal choice of illumination and detection patterns in order to further reduce the data set while preserving the information content.

\section{ACKNOWLEDGMENTS}

This work was partially supported by Cariplo Foundation under Grant N. 20130615 and by the Royal Society International Exchanges 2014/R1.

\section{REFERENCES}

[1] Leff, D. R., Warren, O. J., Enfield, L. C., Gibson, A., Athanasiou, T., Patten, D. K., Hebden, J., Yang, G. Z., and Darzi, A., "Diffuse optical imaging of the healthy and diseased breast: a systematic review.," Breast Cancer Res. Treat. 108(1), 9-22 (2008).

[2] Eggebrecht, A. T., Ferradal, S. L., Robichaux-Viehoever, A., Hassanpour, M. S., Dehghani, H., Snyder, A. Z., Hershey, T., and Culver, J. P., "Mapping distributed brain function and networks with diffuse optical tomography," Nat. Photonics 8(6), 448-454 (2014).

[3] Cherry, S. R., "In vivo molecular and genomic imaging: new challenges for imaging physics.," Phys. Med. Biol. 49(3), R13-R48 (2004).

[4] Ntziachristos, V., "Fluorescence molecular imaging," Annual Review of Biomedical Engineering 8, 1-33 (2006).

[5] Ducros, N., Bassi, A., Valentini, G., Schweiger, M., Arridge, S., and D'Andrea, C., "Multiple-view fluorescence optical tomography reconstruction using compression of experimental data.," Opt. Lett. 36(8), 1377-1379 (2011).

[6] Ducros, N., Bassi, A., Valentini, G., Canti, G., Arridge, S., and D'Andrea, C., "Fluorescence molecular tomography of an animal model using structured light rotating view acquisition.," J. Biomed. Opt. 18, 20503 (2013).

[7] Bassi, A., D'Andrea, C., Valentini, G., Cubeddu, R., and Arridge, S., "Temporal propagation of spatial information in turbid media.," Opt. Lett. 33, 2836-2838 (2008).

[8] Venugopal, V., Chen, J., Lesage, F., and Intes, X., "Full-field time-resolved fluorescence tomography of small animals," Opt. Lett. 35(19), 3189 (2010).

[9] Arridge, S., "Optical tomography in medical imaging," Inverse Problems 15(2), R41-R49 (1999).

[10] Martelli, F., Binzoni, T., Pifferi, A., Spinelli, L., Farina, A., and Torricelli, A., "There's plenty of light at the bottom : statistics of photon penetration depth in random media," Sci. Rep. 6, 27057 (2016).

[11] Cuccia, D. J., Bevilacqua, F., Durkin, A. J., and Tromberg, B. J., "Modulated imaging: quantitative analysis and tomography of turbid media in the spatial-frequency domain.," Opt. Lett. 30(11), 1354-6 (2005).

[12] Joshi, A., Bangerth, W., and Sevick-Muraca, E. M., "Non-contact fluorescence optical tomography with scanning patterned illumination," Opt. Express 14(14), 6516-6534 (2006).

[13] Venugopal, V. and Intes, X., "Adaptive wide-field optical tomography.," J. Biomed. Opt. 18(3), 036006 (2013).

[14] Rudge, T., Soloviev, V., and Arridge, S., "Fast image reconstruction in fluoresence optical tomography using data compression," Optics Letters 35(5), 763-765 (2010).

[15] Duarte, M., Davenport, M., Takhar, D., Laska, J., Sun, T. S. T., Kelly, K., and Baraniuk, R., "Single-Pixel Imaging via Compressive Sampling," IEEE Signal Process. Mag. 25 (2008).

[16] Pian, Q., Yao, R., Zhao, L., and Intes, X., "Hyperspectral time-resolved wide-field fluorescence molecular tomography based on structured light and single-pixel detection," Opt. Lett. 40(3), 431-4 (2015).

[17] Konugolu Venkata Sekar, S., Dalla Mora, A., Bargigia, I., Martinenghi, E., Lindner, C., Farzam, P., Pagliazzi, M., Durduran, T., Taroni, P., Pifferi, A., and Farina, A., "Broadband (600-1350 nm) Time Resolved Diffuse Optical Spectrometer for Clinical Use," IEEE J. Sel. Top. Quantum Electron. 22(3), 406-414 (2015).

[18] Beer, T., "Walsh transforms," Am. J. Phys. 49(5), 466 (1981).

[19] Schweiger, M., Arridge, S. R., and Nissilä, I., "Gauss-Newton method for image reconstruction in diffuse optical tomography," Phys. Med. Biol. 50(10), 2365-2386 (2005). 